\title{
CHROMOSOME NUMBERS IN COMPOSITAE VII: ASTEREAE III ${ }^{1}$
}

\author{
Otto T. Solbrig, loran C. Anderson, Donald W. Kyhos, and Peter H. Raven \\ Department of Botany, University of Michigan, Ann Arbor, \\ Division of Biology, Kansas State University, Manhattan, \\ Department of Botany, University of California, Davis, \\ and \\ Department of Biological Sciences, Stanford University, Stanford, California
}

\section{$\boldsymbol{A} \mathbf{B} \mathbf{S} \mathbf{T} \mathbf{R} \mathbf{A} \mathbf{T}$}

\begin{abstract}
Reports of 129 new chromosome counts are made for the tribe Astereae of Compositae. They are mostly based on determinations of meiotic material, including first counts for one genus and 43 species or subspecies. Counts are now available for more than 63 of the $100-120$ genera and 564 of the approximately 2,000 species in the tribe. Three of every four genera with more than one species counted show more than one chromosome number; 15 genera have species with populations with different numbers. Such variation is very high and indicates the need for more detailed cytotaxonomical study in the group.
\end{abstract}

ThIS IS THE SEVEN'TH of a series of papers dealing with chromosome numbers in Compositae (Raven et al., 1960; Raven and Kyhos, 1961; Ornduff et al., 1963, 1967; Payne, Raven, and Kyhos, 1964; Solbrig et al., 1964) and the third devoted to the tribe Astereae. Since our last work on the tribe, several papers reporting new chromosome numbers for species of Astereae have been published. These reports have tended to confirm the general conclusions and predictions made regarding chromosome number and phylogeny in the tribe Astereae (Solbrig et al., 1964). The reader interested in these topics is referred to that work.

As in previous reports the present combined effort has been arrived at by the authors working largely independently. Unless otherwise indicated the counts have been made from acetocarmine or acetoorceine squashes of microsporocytes in meiosis. Voucher specimens are available for all counts and are deposited at either the Herbarium of the University of California or the Jepson Herbarium, Berkeley, or the Gray Herbarium of Harvard University, unless otherwise indicated.

COMPARISON WITH EARLIER REPORTS-New reports are presented for one genus and 43 species and subspecies indicated in Table 1 by asterisks. The new genus is Archibaccharis from Central America.

Our counts of $n=13, n=18$, and $n=36$ for Aster chilensis subsp. adscendens do not correspond with the previous report of $n=32$

${ }^{1}$ Received for publication 19 September 1968.

The authors are grateful to Denis Breedlove, Angel L. Cabrera, Elias De la Sota, Paul Hutchinson, and Duncan Porter for seeds and fixed materials and to Lee Snyder for technical assistance.

Supported in part by a grant of The National Science Foundation to 0 . T. S. for that taxon (Huziwara, 1958). The count of $n$ $=12$ for Haplopappus racemosus subsp. glomeratus represents a new ploidy level for that taxon as does the count of $n=8$ for $H$. spinulosus subsp. spinulosus. The previous report for Leucelene ericoides was $n=8$ (De Jong and Longpre, 1963; Turner and Flyr, 1966); we found $n=16$ in three different populations. A previous count for Machaeranthera leucanthemifolia was $n=5$ (Raven et al., 1960); we found $n=4$ in two collections, and believe that the plant we examined earlier probably had a supernumerary pair of chromosomes. Our count of $n=8$ for Gymnosperma glutinosum differs from previous reports of $n=7$ for the same species (Solbrig, 1961).

Chromosome nUmbers IN THE TRIBE ASTEREAE-There is now a fairly good understanding of chromosome numbers in the tribe Astereae, particularly in North America. Several mediumsized genera, such as Astranthium (De Jong, 1965), Chrysothamnus (Anderson, 1966) and Gutierrezia (Rüdenberg and Solbrig, 1964), have now been surveyed extensively. More information is needed, however, about the chromosome numbers of the species in the rich assemblages of Astereae of South America, Africa, Australia and New Zealand.

Table 2 lists in synoptical fashion the chromosome numbers in all genera of the tribe for which at least one count is available. The preeminence of $x=9$ as a modal number in the tribe, both at the specific and generic level reported by us previously (Raven et al., 1960; Solbrig et al., 1964) remains unchanged. Likewise, the additional reports since our last paper have tended to confirm the correlation between $x=9$ and certain characteristics such as the woody habit and more mesic environments, correlations that were inter- 
TABLE 1. Original counts of chromosomes in Astereae

A phanostephus arizonicus A. Gray

A phanostephus ramosissimus DC.

Aphanostephus riddellii T. \& G.

A phanostephus skirrobasis (DC.) Trel. var. skirrobasis

A phanostephus skirrobasis (DC.) Trel. var. thalassius Shinners

*Archibaccharis asperifolia (Benth.) Blake

* Archibaccharis torquis Blake

* Aster calendulaceus (Griseb.) Ktze

Aster chilensis Nees subsp. adscendens (Lindl.) Cronq.

Aster exilis Ell.

*Aster eatonii (A. Gray) Howell

*Aster foliaceus Lindl. var. parryi (D. C. Eat.)

A. Gray

* Aster greatai Parish

*Aster marginatus $\mathrm{H}$. B. K.

Aster montevidensis Griseb.

Aster occidentalis (Nutt.) T. \& G.

Aster pauciflorus (Nutt.)

*Aster scopulorum A. Gray

Aster squamatus (Spr.) Hier.

${ }^{*}$ Baccharis salicifolia (R. et P.) Pers.

Baccharis trinervis (Lam.) Pers.

Baccharis sp.

${ }^{*}$ Chaetopappa lyoni (Gray) Keck

Chrysopsis pilosa Nutt.

Chrysopsis villosa (Pursh) Nutt.

Conyza bonariensis (I.) Cronq.

Conyza canadensis (L.) Cronq.

${ }^{*}$ Conyza coulteri Gray

Conyza sp.

Erigeron annuus (L.) Pers.

Erigeron aphanactis (A. Gray) Greene var. aphanactis Cronq.

* Erigeron barbellulatus Greene

Erigeron bellidiastrum Nutt.

Erigeron breweri A. Gray

* Erigeron chrysopsidis A. Gray

*Erigeron chrysopsidis A. Gray var. austinae (Greene) Cronq.
Lincoln Co., N. M., R 19139

Terrell Co., Texas, R 19197

Menard Co., Texas, R 19261

Coke Co., Texas, R 19280

Coke Co., Texas R 19282

Mitchell Co., Texas, R 19285

Galveston Co., Texas, R 19419

Mexico, Mun. of Tenejapa, Chiapas, Br 6893

Mexico, Mun. of Chamula, Chiapas, Br 7151

Uruguay, Riachuelo, Cabrera, 1963 (progeny)

Uinta Co., Wyo., K 5842

Grand Co., Colo., A 2662

White Pine Co., Nev., K 5796

Mexico, Mun. of Pueblo Nuevo, Solistahuacán Chiapas, R 19927

White Pine Co., Nev., Br 5797

Sublette Co., Wyo., $\mathrm{Br} 5855$

Los Angeles Co., Calif., R 16842

Argentina, Prov. Bs. As., La Plata, De la Sota, s.n. (progeny)

Colombia, Bogotá, páramo near Bogotá, Soejarto s.n. (progeny)

Uruguay, Riachuelo, Cabrera s.n. (progeny)

White Pine Co., Nev., Br 5813

Kern Co., Calif., Twisselmann 8450

Harney Co., Ore., R 18436

Elko Co., Nev., R 18527

Brazil, Est. Paraná, Curitiba S 62-44 (progeny)

Argentina, Prov. Jujuy, Dept. Tumbaya Cabrera \& Solbrig 16886 (GH-LP)

Argentina, Prov. La Rioja, Dept. Chilecito, Cabrera \& Solbrig 16679 (GII-LP)

Mexico, Mun. of Jitotol, Chiapas, R 19809

Argentina, Prov. Entre Rios, Colón, Burkart 23547 (progeny)

I.os Angeles Co., Calif., R 19017

Guadalupe Co., Texas, R 19379

Montgomery Co., Texas, R 19441

Grand Co., Colo, A 2651

Coconino Co., Ariz., A 2148

Mexico, Mun. of Pueblo Nuevo, Chiapas, R 19870

Colombia, Dept. Nariño, Porter 1045

Argentina, Prov. Bs. As., Ilavallol, S 62-15 (progeny)

Jamaica, Portland, Porter 1001

Frio Co., Texas, R 19356

Kings Co., Calif., Twisselmann 9097

Costa Rica, Prov. Guanacaste, D. M. Porter 1162

Pottawatomie Co., Kan., R 19484

9 Washoe Co., Nev., R 18413

ca. 40 Alpine Co., Calif., K 5612

9 Apache Co., Ariz., A 2672

9 Major Co., Okla., R 19470

9 Alpine Co., Calif., Br 5626

9 Grant Co., Ore., R 18466

9 Owyhee Co., Idaho, R 18464

$9 \quad$ Malheur Co., Ore., R 19589 
Table 1. Continued

Erigeron compositus Pursh var. discoideus A.Gray Erigeron compositus Pursh var. glabratus Macoun *Erigeron coulteri Porter

Erigeron divergens T. \& G. var. cinereus A. Gray

Erigeron divergens T. \& G. var. divergens

*Erigeron eatonii A. Gray var. eatonii

*Erigeron eatonii A. Gray var. villosus Cronq.

*Erigeron elatior (A. Gray) Greene

*Erigeron fernandezianus (Colla) Solbrig

*Erigeron filifolius Nutt. var. filifolius Cronq.

Erigeron flagellaris A. Gray

Erigeron glabellus Nutt. var. glabellus

*Erigeron linearis (Hook.) Piper

*Erigeron lonchophyllus Hook.

*Erigeron modestus Gray

Erigeron philadelphicus L.

*Erigeron poliospermus Gray var. poliospermus

*Erigeron pumilus Nutt. subsp. concinnoides Cronq. var. concinnoides

*Erigeron pumilus Nutt. ssp. intermedius Cronq. var. gracilior Cronq.

*Erigeron pumilus Nutt. ssp. intermedius Cronq. var. intermedius

*Erigeron pumilus Nutt. subsp. pumilus

*Erigeron pumilus Nutt. (atypical, nearest subsp. intermedius Cronq.)

Erigeron strigosus Muhl. var. beyrichii (F. \& M.) T. \& G.

Erigeron strigosus Muhl. var. strigosus

*Erigeron ursinus D.C. Eat.

Erigeron sp.

* Grindelia decumbens Greene

* Grindelia nana Nutt.

Grindelia discoidea H. \& A.

Grindelia pulchella Dun.

Grindelia sp.

Gutierrezia sarothrae (Pursh) Britt. \& Rusby

Gymnosperma glutinosum Less.

${ }^{*}$ Haplopappus acaulis (Nutt.) Gray

*Haplopappus aff. clementis Blake

Haplopappus ericoides (Less.) H. \& A. subsp. blakei C. B. Wolf

*Haplopappus lanuginosus A. Gray ssp. andersonii (Rydb.) Hall

Haplopappus racemosus (Nutt.) Torr. subsp. glomeratus (Nutt.) Hall

Haplopappus spinulosus (Pursh) DC. subsp. spinulosus

*Haplopappus suffruticosus (Nutt.) A. Gray

Haplopappus validus (Rydb.) Cory subsp. validus
$2 n=58-9 \quad$ Tuolumne Co., Calif., Br 5694

18 Tuolumne Co., Calif., Br 5693.

9 Grand Co., Colo., A 2647

27 I (12 cells) Lincoln Co., N. M., R 19134.

25 I +1 II ( 2 cells $)$

$(23 \mathrm{I}+2$ II 1 ( cell)

$2 n=27 \quad$ Mohave Co., Ariz., R 18971

$2 n=36 \quad$ Mono Co., Calif., R 18555

18 Lassen Co., Calif., R 18424

18 White Pine Co., Nev., Br 5815

9 Adams Co., Idaho, K 18505

9 Grand Co. Colo., A 2648

27 Chile, Masatierra, Juan Fernandez Isl., S 3510

27 Chile, Masafuera, Juan Fernandez Isl., S 3645

18 Grant Co., Ore., R 18481

9 Sherman Co., Ore., R 18387

18 Grand Co., Colo., A 2654

18 Weld Co., Colo., R 19524

9 Yakima Co., Wash., R 18484

$2 n=27 \quad$ Harney Co., Ore., R 18435

9 Tuolumne Co., Calif., Br 5775

27 Sutton Co., Texas, R 19252

27 Menard Co., Texas, K 19262

9 Canyon Co., Idaho, R 19572

$9 \quad$ Harney Co., Ore., R 18433

$9 \quad$ Clark Co., Nev., R 18917

9 Mohave Co., Ariz., R 19102

9 Owyhee Co., Idaho, R 18465

18 Fremont Co., Idaho, R 19559

$9 \quad$ Grant Co., Ore., R 18482

$9 \quad$ Weld Co., Colo., R 19519

9 Laramie Co., Wyo., R 19528

$2 n=27$ (triploid) Storey Co., Nev., R 18545

$\begin{aligned} 27 & \text { Riley Co., Kan., R 19480 } \\ 27 & \text { Montgomery Co., Texas, R 19439 } \\ 27 & \text { Hunt Co., Texas, R 19446 } \\ 9 & \text { Grand Co., Colo., A 2664 } \\ 27 & \text { Brewster Co., Texas, S 3194 } \\ 6 & \text { Montezuma Co., Colo., A 2678 } \\ 6 & \text { Grant Co., Wash., R 18488 } \\ 12 & \text { Argentina, Prov. Cordoba, Dept. Santa } \\ & \text { Maria, Cabrera \& Solbrig 16551. (GH-LP) } \\ 6 & \text { Argentina, Prov. Catamarca, Dept. Tino- } \\ & \text { gasta, Cabrera \& Solbrig 16748. (GH-LP) } \\ 6 & \text { Terrell Co., Texas, R 19196 } \\ 4 & \text { Menard Co., Texas, K 19274 } \\ 8 & \text { Mexico, Mun. of Comitán, Chiapas, Br } 8329 \\ 18 & \text { Elko Co., Nev., R 18530 } \\ 18 & \text { Alpine Co., Calif., Br 5614 } \\ & \text { Park Co., Wyo., S 3116 } \\ 9 & \text { Los Angeles Co., Calif., Thompson } 1819 \\ 9 & \text { Valley Co., Idaho, R 18514 } \\ 12 & \text { Kern Co., Calif., Twisselmann 10169 } \\ 8 & \text { Weld Co., Colo., R 19521 } \\ 9 & \text { White Pine Co., Nev.,Br.5817 } \\ 5 & \text { Garza Co., Texas, R 19298 } \\ 5 & \text { Tarrant Co., Texas, R 19449 }\end{aligned}$


TABLE 1. Continued

*Haplopappiüs whitneyi A. Gray

Heterotheca latifolia Buckl. var. macgregoris

Wagenknecht

*Lessingia lemmonii A. Gray var. ramulosissima

(A. Nels.) Ferris

Leucelene ericoides (Torr.) Greene

*Machaeranthera bigelovii (Gray) Greene

*Machaeranthera commixta Greene

Machaeranthera grindelioides (Nutt.) Shinners

Machaeranthera leucanthemifolia (Greene) Greene

* Machaeranthera shastensis Gray var. montana

(Greene) Cronq.

Machaeranthera tanacetifolia (Kunth) Nees

Monoptilon bellidiforme T. \& G. ex Gray

Psila sp.

*Solidago ciliosa Greene

*Solidago spectabilis (D. C. Eat.) Gray

Xanthisma texanum DC.

Xylorhiza glabriuscula Nutt.
Kern Co., Calif., Twisselmann 10178

Gillespie Co., Texas, R 19337

Inyo Co., Calif., Hill 47937

Mohave Co., Ariz., R 19101

Yavapai Co., Ariz., R 19108

Torrance Co., N. M., R 19131

Yavapai Co., Ariz., R 19105

Fremont Co., Idaho, R 19556

White Pine Co., Nev., Br 5795

Uinta Co., Wyo., Br 5843

Sweetwater Co., Wyo., R 19542.

Nye Co., Nev., Br 5665

Clark Co., Nev., K 18908

Alpine Co., Calif., Br 5613

Weld Co., Colo., R 19518

Midland Co., Texas, R 19216

Clark Co., Nev., R 18891

Nye Co., Nev., K 18954

Argentina, Prov: Jujuy, Cabrera \& Solbrig 17030. (GH-LP)

Grand Co., Colo., A 2646

Owyhee Co., Idaho, R 19575

Midland Co., Texas, R. 19217

Gillespie Co., Texas, R 19338

Garza Co., Texas, R 19299

Albany Co., Wyo., R 19536

Sweetwater Co., Wyo., R 19546

a Collection numbers preceded by $\mathbf{A}$ are Anderson; Br, Breedlove; K, Kyhos; R, Raven; S, Solbrig.

TABLE 2. Chromosome numbers in genera of Compositae

\begin{tabular}{|c|c|c|c|c|c|c|c|c|}
\hline Genus & $\begin{array}{l}\text { Approx. } \\
\text { \# sp. }\end{array}$ & $\begin{array}{l}\text { \# sp. } \\
\text { counted }\end{array}$ & Geog. distrib. & & Habit & Chrom. \#'s & $\begin{array}{c}\text { Type of } \\
\text { var:a }\end{array}$ & $\begin{array}{c}\text { Intraspec. } \\
\text { variation }\end{array}$ \\
\hline Acamptopappus & 2 & 2 & N. America & $\because$ & $\mathbf{S}$ & 9 & & no \\
\hline Achaetogeron & 7 & 3 & N. America & & $\mathbf{P}$ & 9,27 & $\mathbf{P}$ & no \\
\hline Amellus & 10 & 1 & S. Africa & & $\mathbf{A}, \mathbf{P}$ & 8 & & no \\
\hline Amphiachyris & 2 & 1 & N. America & & A & 5 & & no \\
\hline Amphipappus & 1 & 1 & N. America & & $\mathrm{S}$ & 9 & & no \\
\hline Archibaccharis & 18 & 2 & N. America & & $\mathbf{S}$ & 9 & & no \\
\hline Aphanostephus & 5 & 5 & N. America & & $\mathbf{A}, \mathbf{P}$ & $3,4,5$ & A & no \\
\hline Aster & $250^{+}$ & 102 & cosm. & & $A, P, S$ & $\begin{array}{c}5,7,8,9,10 \\
16,18,19 \\
23,24,27 \\
32,33,36\end{array}$ & $\mathbf{A}, \mathbf{P}$ & yes \\
\hline Asteromea & 2 & 2 & Asia & & $\mathbf{P}$ & 9 & & no \\
\hline Astranthium & 12 & 12 & N. America & & $\mathbf{A}, \mathbf{P}$ & $\begin{array}{l}3,4,5,6,8 \\
9,10,12,18\end{array}$ & $\mathbf{A}, \mathbf{P}$ & no \\
\hline Baccharis & $350^{+}$ & 18 & N., S. America & & $\mathrm{P}, \mathrm{S}$ & 9,18 & $\mathbf{P}$ & no \\
\hline Bellidiastrum & 1 & 1 & Europe & & $\mathbf{P}$ & 9 & & no \\
\hline Bellis & 5 & 3 & Europe & & A, $\mathrm{P}$ & 9 & & no \\
\hline Bellium & 5 & 1 & Europe & & $\mathbf{A}, \mathbf{P}$ & 9 & & no \\
\hline Benitoa & 1 & 1 & N. America & & $\mathrm{A}$ & 5 & & no \\
\hline Boltonia & 3 & 3 & N. America & & $\ddot{P}$ & $9,18,27$ & $\mathbf{P}$ & yes \\
\hline Brachycome & 50 & 3 & Australia & & $A, P$ & $9,18,27$ & $\mathrm{P}$ & no \\
\hline Bradburia & 1 & 1 & N. Amerida & & A & 3 & & no \\
\hline Calotis & 30 & 2 & Australia & & $\mathbf{P}$ & 7,8 & $\mathbf{A}$ & no \\
\hline Celmisia & 35 & 2 & N. Z., S. America & & $\mathbf{P}$ & 9 & & no \\
\hline Chaetopappa & 15 & 2 & N. America & & $A, P$ & $8,9,16$ & $\mathbf{A}, \mathbf{P}$ & yes \\
\hline Charieis & $\mathbf{1}_{\text {in }}$ & 1 & S. Africa & & A & 5 & & no \\
\hline
\end{tabular}


TABLE 2. Continued

\begin{tabular}{|c|c|c|c|c|c|c|c|}
\hline Genus & $\begin{array}{l}\text { Approx. } \\
\text { \# sp. }\end{array}$ & $\begin{array}{l}\text { \# sp. } \\
\text { counted }\end{array}$ & Geog. distrib. & Habit & Chrom. \#'s & $\begin{array}{l}\text { Type of } \\
\text { var." }\end{array}$ & $\begin{array}{c}\text { Intraspec. } \\
\text { variation }\end{array}$ \\
\hline Chrysopsis & 20 & 9 & N. America & $\mathbf{A}, \mathbf{P}, \mathbf{S}$ & $4,5,9,12,18$ & $\mathbf{A}, \mathbf{P}$ & yes \\
\hline Chrysothamnus & 14 & 14 & N. America & $\mathbf{S}$ & $9,18,36$ & $\mathbf{P}$ & yes \\
\hline Conyza & 50 & 15 & cosm. & A, $\mathbf{P}$ & 9,27 & $\mathrm{P}$ & no \\
\hline Coreothrogyne & 3 & 2 & N. America & $\mathbf{P}$ & 5 & & no \\
\hline Darwiniothamnus & 2 & 2 & Galápagos & $\mathbf{S}$ & 9 & & no \\
\hline Dichaetophora & 1 & 1 & N. America & A & 3 & & no \\
\hline Dichrocephala & 5 & 1 & Africa, Asia & $\mathbf{A}$ & 9 & & no \\
\hline Erigeron & $200^{+}$ & 81 & cosm. & $\mathbf{A}, \mathbf{P}, \mathbf{S}$ & $9,18,27,32,36$ & $\mathbf{A}, \mathbf{P}$ & yes \\
\hline Eastwoodia & 1 & 1 & N. America & S & 9 & & no \\
\hline Erodiophyllum & 1 & 1. & Australia & $\mathbf{S}$ & 8 & & no \\
\hline Felicia & 50 & 7 & S. Africa & $\mathrm{A}, \mathrm{P}$ & $6,8,9$ & $\mathbf{A}$ & yes \\
\hline Grangea & 2 & 1 & Africa, Asia & $\mathbf{P}$ & 9 & & no \\
\hline Greenella & 3 & 1 & N. America & $\mathbf{A}, \mathbf{P}$ & 4 & & no \\
\hline Grindelia & 50 & 22 & N., S. America & $P, S$ & 6,12 & $\mathbf{P}$ & yes \\
\hline Gutierrezia & 19 & 13 & N., S. America & $\mathrm{A}, \mathbf{P}, \mathbf{S}$ & $\begin{array}{c}4,8,12,16 \\
20,28\end{array}$ & $\mathbf{P}$ & yes \\
\hline Gymnosperma & 1 & 1 & N. America & $\mathbf{S}$ & 7,8 & A & yes \\
\hline Haplopappus & 180 & 38 & N., S. America & $A, P, S$ & $\begin{array}{c}2,3,4,5,6,7 \\
\quad 8,9,12,45\end{array}$ & $\mathbf{A}, \mathbf{P}$ & yes \\
\hline Heteropappus & 5 & 3 & Asia & $\mathbf{A}, \mathbf{P}$ & 18 & & no \\
\hline Heterotheca & 8 & 8 & N. America & $\mathbf{A}, \mathbf{P}$ & 9,18 & $\mathbf{P}$ & no \\
\hline Lagenophora & 17 & 1 & S. America, S. Pacific & $\mathbf{P}$ & 9 & & no \\
\hline Lessingia & 7 & 7 & N. America & $\mathbf{A}$ & $\overline{5}$ & & no \\
\hline Leucelene & 1 & 1 & N. America & $\mathbf{P}$ & 8,16 & $\mathbf{P}$ & no \\
\hline Machaeranthera & $30^{+}$ & 17 & N. America & $\mathbf{A}, \mathbf{P}$ & $4,5,6,9$ & A & yes \\
\hline Microglossa & 9 & 3 & Africa, Asia & $\mathbf{S}$ & 9 & $\mathbf{P}$ & no \\
\hline Monoptilon & 2 & 2 & N. America & $\mathbf{A}$ & 8,27 & $\mathbf{A}, \mathbf{P}$ & no \\
\hline Myriactis & 5 & 2 & Asia, Africa & A & 18 & & no \\
\hline Nidorella & 18 & 4 & Africa & $\mathbf{S}, \mathbf{P}$ & 9 & & no \\
\hline Olearia & 90 & 3 & S. Pacific & $\mathbf{T}, \mathbf{S}$ & 9,54 & $\mathbf{P}$ & no \\
\hline Olivaea & 2 & 2 & N. America & A & 6 & & no \\
\hline Petradoria & 2 & 2 & N. America & $\mathbf{S}$ & 9,18 & $\mathbf{P}$ & no \\
\hline Psiadia & 30 & 1 & Africa & $\mathrm{S}$ & 9 & & no \\
\hline Psila & 10 & 2 & S. America & $\mathbf{S}$ & 9 & & no \\
\hline Rhynchosperma & 1 & 1 & Asia & $\mathbf{S}$ & 9 & & no \\
\hline Solidago & 100 & 80 & N., S. America & $\mathrm{S}$ & $9,18,27$ & $\mathbf{P}$ & yes \\
\hline Tetramolopium & 7 & 1 & S. Pacific & $\mathbf{P}$ & 7,9 & $\mathbf{A}$ & yes \\
\hline Townsendia & 21 & 20 & N. America & $\mathbf{A}, \mathbf{P}$ & $9,10,15,18,27$ & $\mathbf{A}, \mathbf{P}$ & yes \\
\hline Vanclevea & 1 & 1 & N. America & $\mathbf{P}$ & 9 & & no \\
\hline Vittadinia & 6 & 1 & S. America, Australia & $\mathbf{P}, \mathbf{S}$ & 9 & & no \\
\hline Xanthisma & 1 & 1 & N. America & A & 4 & & no \\
\hline Xanthocephalım & 8 & 5 & N. America & $\mathbf{A}, \mathbf{P}, \mathbf{S}$ & 4,6 & A & no \\
\hline
\end{tabular}

s Type of variation: A, aneuploidy; P, polyploidy.

preted by us as an indication that $x=9$ is probably the ancestral basic number in the tribe Astereae.

Of the 63 genera that have been counted in the tribe, almost half (30) show variation in the chromosome number. Disregarding the 21 genera for which only a single species has been counted, three out of every four genera remaining show variation in chromosome number. This is a very high degree of variation.

The source of this variation is either polyploidy or aneuploidy. Judging from the chromosome numbers and from observations in those cases where the situation was more carefully investigated, the chromosome variation is due to polyploidy in 14 genera, to aneuploidy in 8 and to a combination of polyploidy and aneuploidy in another 8. More remarkable still is the fact that in 15 genera, more than one chromosome number has been associated with the same species. Although some of these reports may reflect errors in the reporting of the chromosome number or identification of the species, in most cases the chromosomal variation has been confirmed repeatedly. In certain cases some of the chromosomal variation has been associated with subspecific taxa as in Chrysothamnus (Anderson, 1966). In other cases it has not. However in most instances the situation has not been investigated beyond the reporting of the different chromosome number. As the investigations with the Haplopappus gracilis-Haplopappus ravenii species pair 
have shown (Jackson, 1962), careful study of infraspecific variation can be very rewarding in providing detailed records of the process of evolution.

Certain large and taxonomically difficult genera, such as Aster and Erigeron, are particularly notable for the chromosome number variation they exhibit. Other genera such as Chrysothamnus and the genera of the subtribe Baccharidineae are remarkably uniform. The reason for this discrepancy is as yet unknown.

\section{LITERATURE CITED}

Anderson, L. C. 1966. Cytotaxonomic studies in Chrysothamnus (Astereae, Compositae). Amer. J. Bot. 53: 204-212.

De JonG, D. C. D. 1965. A systematic study of the genus Astranthium. Publ. Mus. Mich. State Univ. Biol. Ser. 2: 429-528.

studies in Mexican Compositae. Rhodora 65: 226-240.

Huzrwara, Y. 1958. Karyotype analysis in some genera of Compositae V. The chromosomes of American Aster species. Jap. J. Genet. 33: 129-137.

$\mathrm{J}_{A C K S O N}, R$. 1962. Interspecific hybridization in the genus Haplopappus and its bearing on chromosome evolution in the Blepharodon section. Amer. J. Bot. 49: 119-132.
Ornduff, R., T. Mosquin, D. W. Krhos, and P. H. Raven. 1967. Chromosome numbers in Compositae. VI. Senecioneae II. Amer. J. Bot. 54: 205-213.

- , P. H. Raven, D. W. Kyhos, and A. R. KruckeberG. 1963. Chromosome numbers in Compositae III. Senecioneae. Amer. J. Bot. 50: 131-139.

PAYNe, W. W., P. H. Raven, and D. W. Kyhos. 1964. Chromosome numbers in Compositae IV. Ambrosieae. Amer. J. Bot. 51 : 419-424.

Raven, P. H., and D. W. Kyhos. 1961. Chromosome numbers in Compositae II. Helenieae. Amer. J. Bot. 48: 842-850.

- , O. T. Solbrig, D. W. Kyhos, and R. SNow. 1960. Chromosome numbers in Compositae $I$. Astereae. Amer. J. Bot. 47: 124-132.

Rüdenberg, L., and O. T. Solbrig. 1964. Chromosome number and morphology in the genus Gutierrezia (Compositae-Astereae). Phyton 20: 199-204.

SolbriG, O. T. 1961. Note on Gymnosperma glutinosum (Compositae-Astereae). Leafl. West. Bot. 9: 147-150.

- , I. C. Anderson, D. W. Kyhos, P. H. Raven, AND L. Rüdenberg. 1964. Chromosome numbers in Compositae V. Astereae II. Amer. J. Bot. 51: $513-519$.

Turner, B. L., AND D. Flyr. 1966. Chromosome numbers in the Compositae. $X$. North American species. Amer. J. Bot. 53: 24-33. 